Trauma Berufskrankh 2015 • 17[Suppl 1]:178-183 DOI 10.1007/s10039-014-2128-9

Online publiziert: 22. Januar 2015

c) Springer-Verlag Berlin Heidelberg 2015
S. Drisch ${ }^{1}$ S. Simmel ${ }^{1} \cdot$ V. Bühren ${ }^{1}$ R. Mödl ${ }^{2}$

${ }^{1}$ Abteilung für BG-Rehabilitation, BG-Unfallklinik Murnau

${ }^{2}$ Orthopädietechnik, BG-Unfallklinik Murnau

\title{
Hilfsmittelversorgung bei Kindern und Jugendlichen
}

\section{Beispiel angeborene Longitudinaldefekte und traumatische Amputationen der unteren Extremität}

Kinderchirurgen, Kinderorthopäden, Onkologen, die technische Orthopädie sowie Physio- und Ergotherapeuten haben die z. T. sehr schwierige Aufgabe, einem Kind mit angeborenem oder erworbenem körperlichem Schaden eine optimale Behandlung und Versorgung zukommen zu lassen. Besondere Herausforderungen stellen das Wachstum des Kindes und gelegentlich auch die Eltern, die sich um die Zukunft ihres Kindes sorgen, dar. Im Vordergrund steht deshalb eine optimale Ver- sorgung mit dem Anspruch, die Lebensqualität und Unabhängigkeit des heranwachsenden Kindes so gut wie möglich wiederherzustellen und zu unterstützen. Medizinisch sind jedoch auch hier Grenzen gesetzt, und die Rahmenbedingungen machen die Versorgung des Heranwachsenden zu einer großen Herausforderung.

Der Kostendruck, welcher unsere Entscheidungen nicht beeinflussen sollte, ist nicht zu vernachlässigen. Fallpauschalen vs. einer evtl. komplikationsreichen Am- putation führen $\mathrm{zu}$ einem erheblichen Entscheidungsdruck.

An 2 Fallbeispielen soll aufgezeigt werden, welche Möglichkeiten die technische Orthopädie und die interdisziplinäre $\mathrm{Zu}$ sammenarbeit bieten.

\section{Ätiologie/Epidemiologie}

Die Inzidenz kindlicher Amputationen verteilt sich auf $60 \%$ bei angeborenen Defekten (1:3500, - Abb. 1) und 40\% erwor-

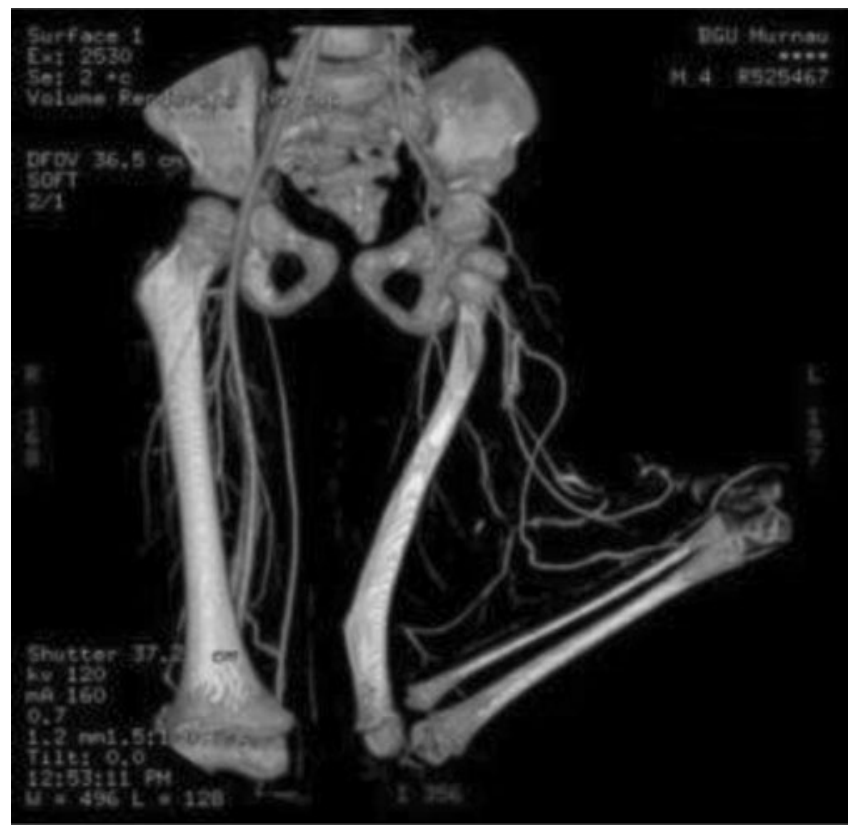

Abb. 1 <-Jähriger mit angeborenem Defekt vor der Amputation

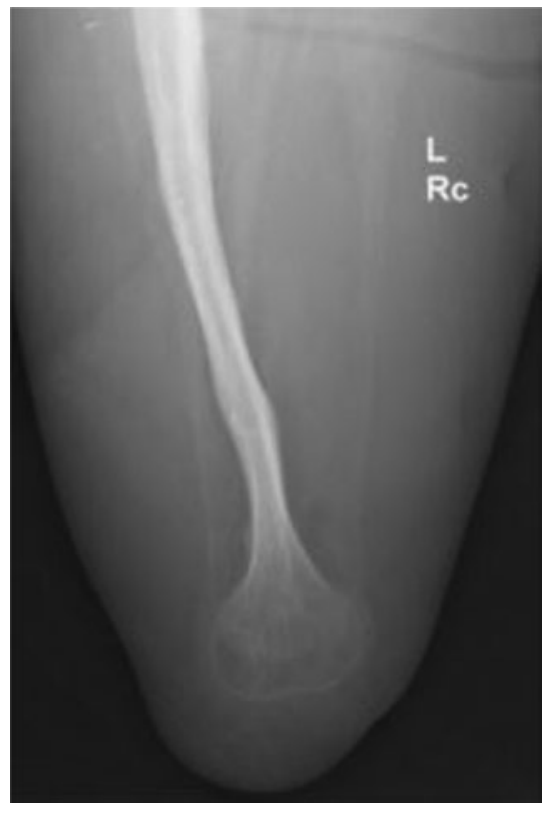

Abb. $2 \Delta$ Wachstumsrückstand in Länge und Breite nach Amputation im Alter von 13 Jahren 

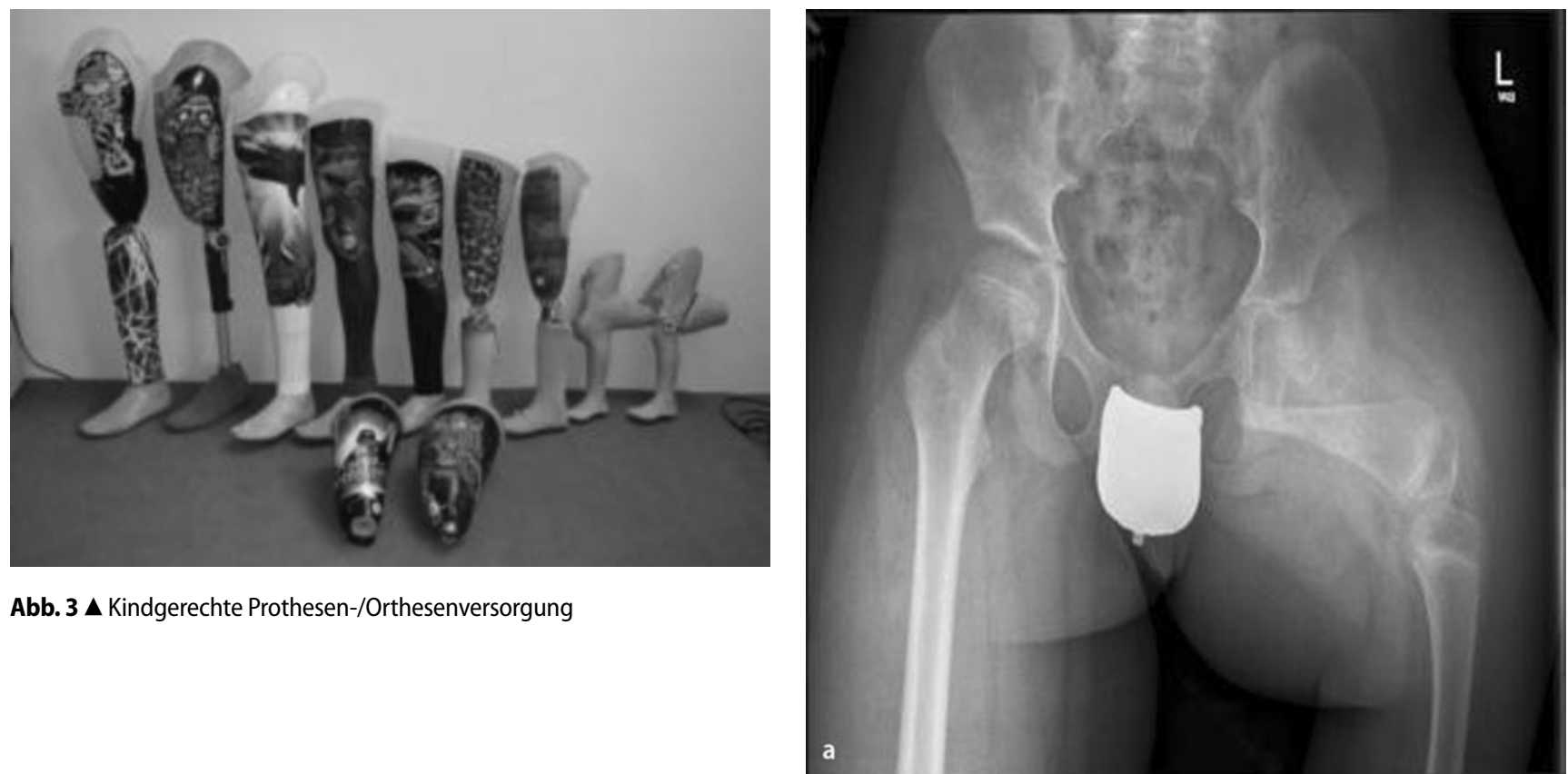

Abb. $3 \Delta$ Kindgerechte Prothesen-/Orthesenversorgung
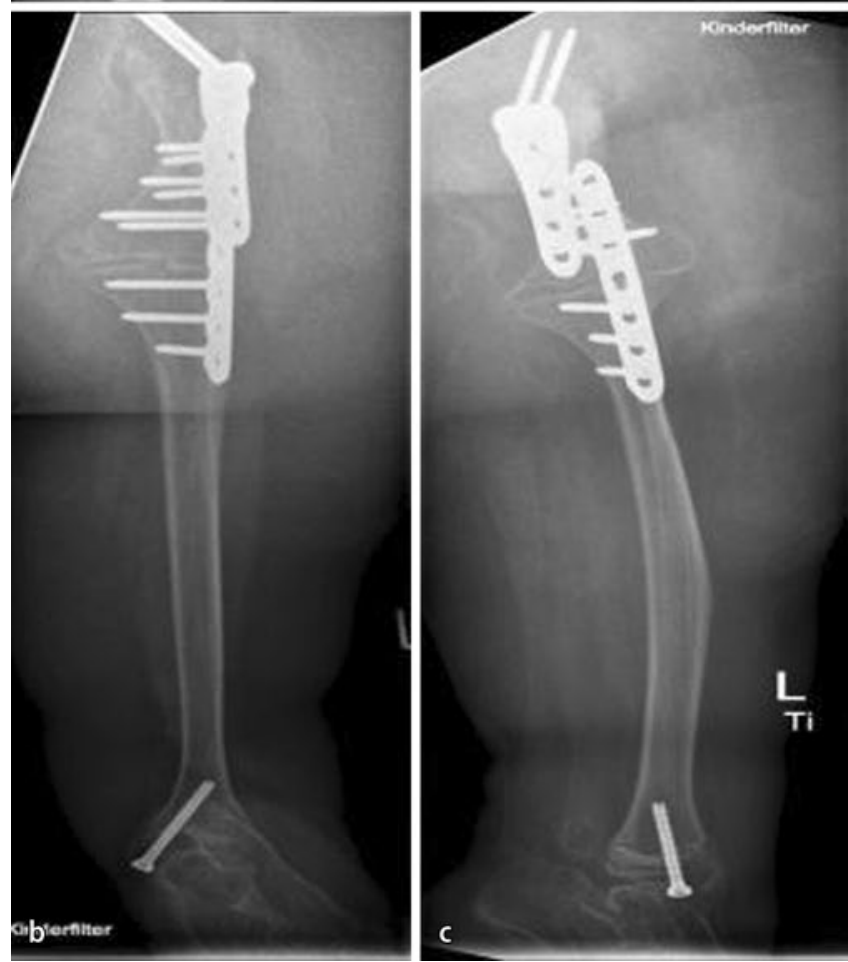

bene Amputationen [2]. Etwa 60\% der angeborenen Gliedmaßenfehlbildungen betreffen die oberen und 30\% die unteren Extremitäten [4].

Die erworbenen Amputationen lassen sich in die Gruppen der traumatischen (einschließlich thermischen) Amputationen, der tumorbedingten Amputationen und der Amputation nach Infektion und Osteomyelitis unterteilen [3]. Sowohl in der Tumorchirurgie als auch bei den traumatischen Amputationen sind fast ausschließlich Jungen und hier die untere häufiger als die obere Extremität betroffen ([1], S. 74).

\section{Besonderheiten}

Der sog. Kindermalus setzt uns Grenzen in der postoperativen Versorgung nach traumatischer Amputation. Die größten Nachteile liegen darin, dass der Wachs- tumsrückstand umso stärker ist, je früher der Verlust des Röhrenknochens auftritt bzw. je höher die Amputation lokalisiert ist. Der Wachstumsrückstand zeigt sich sowohl in der Länge als auch in der Breite (- Abb. 2). Analoge Überlegungen gelten auch für die angeborenen Defekte (• Abb. 1; [1], S. 74).

In - Abb. 3 ist der Versuch dargestellt, ein Kind mit angeborenem Defekt (• Abb. 2, 4) im Zeitraum seines Wachs- 
tums mit Orthesen/Prothesen zu versorgen, um dem drohenden Wachstumsrückstand entgegenzuwirken und das Kind in seiner Mobilität zu unterstützen. Die Prothesen wurden etwa jährlich erneuert und entsprechend den vorhandenen Gliedmaßen und Weichteilen angepasst.

Weitere Nachteile könnten aus der unterschiedlichen Wachstumspotenz der Epiphysenfugen resultieren, wodurch es zu Achsabweichungen kommen kann. Zudem wachsen Narbenkeloide nicht mit, was u. U. zu Kontrakturen führt. Auch ein Nichterreichen einer Endbelastung des Stumpfs kann den Wachstumsrückstand beeinflussen, denn diese Endbelastung stimuliert in der Regel das Wachstum, und Fehlstellungen können prothetisch korrigiert werden ([1], S. 293 ff).

Im Gegensatz zu diesen Nachteilen gibt es auch einen sog. Kinderbonus, der darin liegt, dass die Gewebe noch elastischer sind, die Durchblutung besser ist und Wunden schneller abheilen. Zudem empfinden Kinder, die vor der Pubertät amputiert werden, keinen Phantomschmerz [3].

\section{Kongenitaler Longitudinaldefekt - Fallbeispiel 1}

In diesem Beispiel soll anhand des Verlaufs dargestellt werden, wie gut die $\mathrm{Zu}$ sammenarbeit zwischen Techniker und Kliniker sowie den unterschiedlichen Fachabteilungen funktioniert und welchen Verlauf die Versorgung nahm. Im Endergebnis zeigte sich eine hohe Zufriedenheit des Kindes und der Eltern, mit einem deutlichen Zugewinn an Lebensqualität im Sinne von Mobilität und Teilhabe.

Versorgt werden sollte ein 9-jähriger Junge mit angeborenem proximalem Femurdefekt links ( $\mathbf{C}$ Abb.4a), welcher zu den Longitudinaldefekten zählt. Nach Kniegelenkarthrodese, Epiphyseodese des linken Innenknöchels und hüftgelenknaher Umstellungsosteotomie (- Abb. 4b,c) im Jahr 2012 zeigten sich abschließend noch ein Rotationsfehler des linken Unterschenkels und ein konsekutiver Wundinfekt mit MRSA (methicillinresistenter Staphylococcus aureus). Nach Eradikation des Keims erfolgte eine Metallentfernung mit der intraoperati-

Trauma Berufskrankh 2015 • 17[Suppl 1]:178-183 DOI 10.1007/s10039-014-2128-9

(c) Springer-Verlag Berlin Heidelberg 2015

\section{S. Drisch ·S. Simmel · V. Bühren · R. Mödl \\ Hilfsmittelversorgung bei Kindern und Jugendlichen. Beispiel angeborene Longitudinaldefekte und traumatische Amputationen der unteren Extremität}

\section{Zusammenfassung}

Problematik. Die Hilfsmittelversorgung nach Amputationen im Kindesalter ist eine Herausforderung für alle medizinischen Fachabteilungen. Individuelle Versorgungen durch teambezogene Entscheidungsfindungen und ganzheitliche Behandlungsstrategien sind in diesem Zusammenhang unabdingbar. Häufige Anpassungen und mehrfache Neuversorgungen sind aufgrund des nicht abgeschlossenen Wachstums des Kindes und den damit steigenden Ansprüchen an das Hilfsmittel keine Seltenheit.

Ziel der Hilfsmittelversorgung. Es gilt, den Körperschaden so gut als nur möglich auszugleichen, um eine Integration in den Alltag gewährleisten zu können.
Resümee. Für Heranwachsende stellt die Teilhabe am sozialen Leben mit einer solchen Behinderung eine nicht unerhebliche physische und psychische Belastung dar. Für uns sollte es außer Frage stehen, dass alle Mittel zur Verfügung gestellt werden, um es den Kindern und Jugendlichen so einfach wie möglich zu machen, sich an ein solches Hilfsmittel zu gewöhnen und es eigenverantwortlich im Alltag benutzen zu können.

\section{Schlüsselwörter}

Kinder - Traumatische Amputation .

Kongenitaler Defekt · Orthese · Prothese

\section{Provision of orthotic devices in children and adolescents. Exemplified by congenital longitudinal defects and traumatic amputations of the lower extremities}

\section{Abstract}

Background. The provision of orthotic devices following amputation in childhood is a challenge for all medical specialist departments. Individual treatment and constructions by team-related decision-making are essential in this context. Frequent adjustments and new fittings are not uncommon due to the incompleted growth phase of the child and the associated increasing demands on the device.

Provision of orthotic devices. The aim is to compensate the body damage as far as possible to enable the child to become reintegrated into the activities of daily living.
Conclusion. The participation in normal social life for adolescents with such a disability involves a significant degree of physical and psychological stress. There should be little doubt that all funds necessary are made available to make it as easy as possible for children and young people to get used to the devices so that they can be used independently in everyday life.

\section{Keywords}

Children · Traumatic amputation · Congenital defect · Orthosis · Prosthesis ven Feststellung einer eingeschränkten, eher weichen Knochenqualität, aufgrund der jahrelangen Entlastung ohne orthetische/prothetische Versorgung. In gleicher Sitzung wurden die keloidartigen Narben korrigiert. $\mathrm{Zu}$ diesem Zeitpunkt stellte sich für die Orthopädietechnik die Frage nach der Belastungsmöglichkeit und dem Risiko einer Fraktur aufgrund der beschriebenen weichen Knochenqualität.

Trotz der erwähnten Bedenken wurde mit der orthetischen/prothetischen Versorgung begonnen, denn man erwartete sich durch die zunehmende Belastung eine Verbesserung der Knochenqualität, da durch eine adäquate axiale Belastung indirekt das Wachstum stimuliert und somit der Stoffwechsel und die Remineralisierung angeregt werden können. Der Ablauf der Versorgung gestaltete sich wie in - Abb. 5 dargestellt.

Erfreulicherweise war es kein Problem, den 9-jährigen Jungen zu einer guten Mitarbeit während des Gipsabdrucks zu bewegen. Nicht immer stellt sich dies so einfach wie in unserem Beispiel dar, und die Versorgung ist daher häufig mit 

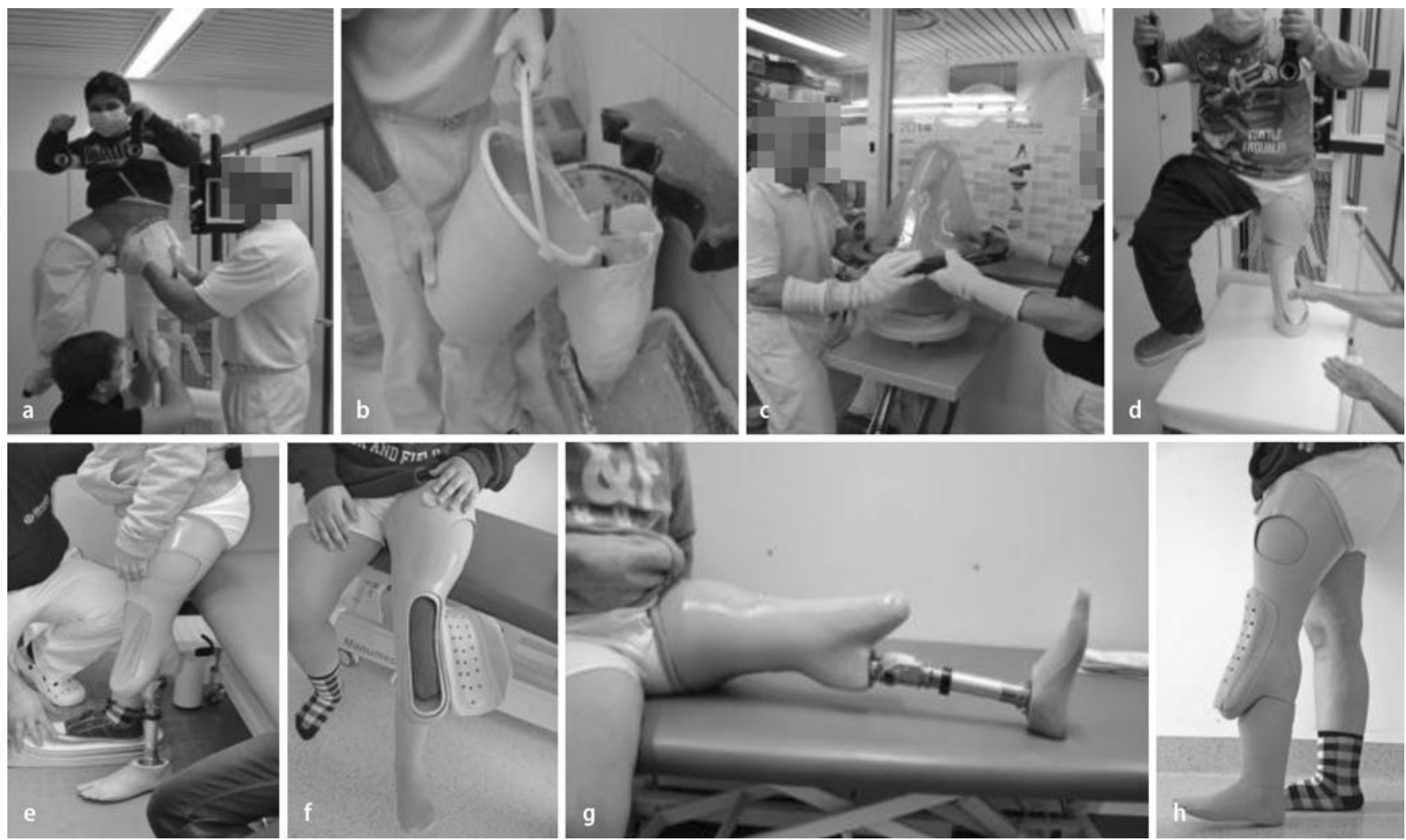

Abb. $5 \Delta$ Fallbeispiel 1, Ablauf der orthetischen/prothetischen Versorgung: a Gipsabdruck, b Erstellen eines sog. Positivs, c sog. Tiefziehverfahren über das Positiv und Vakuumierung, $\mathbf{d}$ Erstellen eines Probeschafts mit Weichwandinnentrichter, e Anpassung der Orthese und Ausgleich der Gegenseite, $\mathbf{f}$ Fertigstellung der Orthese/Prothese, $\mathbf{g}, \mathbf{h}$ Seitansicht ohne (g) und mit Kosmetik (h)

einem nicht unerheblichen Zeitaufwand verbunden.

Im Anschluss an die Versorgung wurden intensive Übungen in der Gehschule sowie eine sog. instrumentelle Ganganalyse und mehrere Videoaufnahmen zur Kontrolle des Gangbildes durchgeführt. Durch diese Analysen konnten zielgerichtet das Gleichgewicht und die Belastung des orthesenversorgten Beins trainiert werden. Mit dem Abschluss des Aufenthalts konnte der Junge an einem Handstock in der Ebene gehen, Schräge und Treppe sowie kleinere Hindernisse überwinden. Spielerisch wurde die Koordination mit z. B. Fußballspielen trainiert.

\section{Traumatische Hemipelvektomie}

\section{Im Rahmen eines Arbeitsunfalls -} Fallbeispiel 2

Aus Abb. 6 geht die Versorgung hervor, welche bei einem jetzt 26-jährigen Patienten durchgeführt wurde, der eine trau- matische Hemipelvektomie im Rahmen eines Arbeitsunfalls erlitt (ब Abb. 6a).

Die Schwierigkeit bei diesem Patienten war das abdominelle Trauma mit einem künstlichen Darmausgang. Die Darmbeinschaufel, der Sitzbeinast und der Schambeinast rechts konnten z. T. erhalten werden, was eine noch gute Ausgangssituation für die orthopädietechnische Versorgung mit einem Beckenkorb bietet. Die Einzelteile der prothetischen Versorgung setzten sich zusammen aus einem Karbonrahmen (Außenschaft), einem Helixhüftsystem, einem mikroprozessorgesteuerten Kniegelenk und einem Karbonfederfuß. Wie in $\square$ Abb. 6b-d ersichtlich konnte eine zufriedenstellende Versorgung des jungen Patienten erfolgen.

Im klinischen Alltag zeigte sich jedoch, dass auf diese Art versorgte Patienten zusätzlich auf einen Rollstuhl angewiesen sind. Denn das Gehen mit einem Beckenkorb erfordert einen erheblichen Kraftund Energieaufwand, und längere Gehstrecken sind in der Regel nicht gut zu be- wältigen. Oft kommen weitere Hilfsmittel wie Unterarmgehstützen oder Handstöcke zum Einsatz. Die Praxis zeigt uns eine maximale Tragedauer von etwa $2-4 \mathrm{~h}$ täglich. Durch das Silikonmaterial kommt es zu einer erheblichen Schweißneigung, wodurch immer ein kleines Risiko der Hautirritation bis hin zu Hautreizungen, Wundgeschwüren und nicht selten zu Follikulitiden gegeben ist.

\section{Nach Überrolltrauma mit erheblichem Weichteilschaden - Fallbeispiel 3}

Bei dem in $\square$ Abb. 7 dargestellten Fall handelte es sich um eine 13-Jährige nach Hemipelvektomie im Jahr 2012 mit erheblichen zusätzlichen Komplikationen durch eine hypertrophe Narbenbildung nach Überrolltrauma und großem Weichteildefekt.

Zum Teil waren die Areale spalthautoder lappengedeckt und zeigen eine gute Konditionierung, jedoch ohne bisherige komplette Ausreifung. Bei der klini- 


\section{Spezielle Verletzungsformen und Rehabilitation}
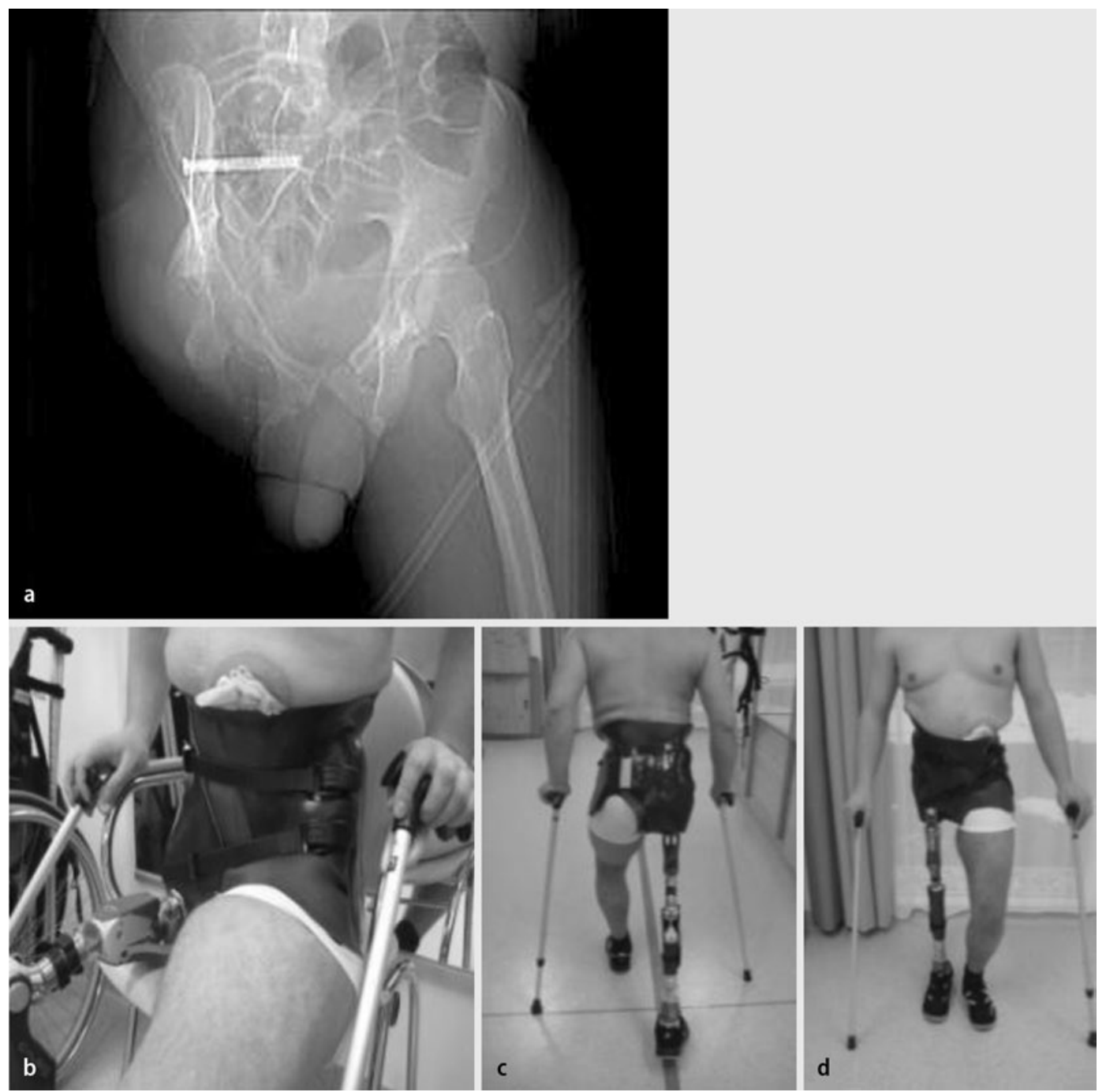

Abb. $6<$ Fallbeispiel 2, männlicher Patient, 26 Jahre, a traumatische Hemipelvektomie, $\mathbf{b}, \mathbf{c}, \mathbf{d}$ Beckenkorb mit Helixhüftsystem und mikroprozessorgesteuertem Kniegelenk
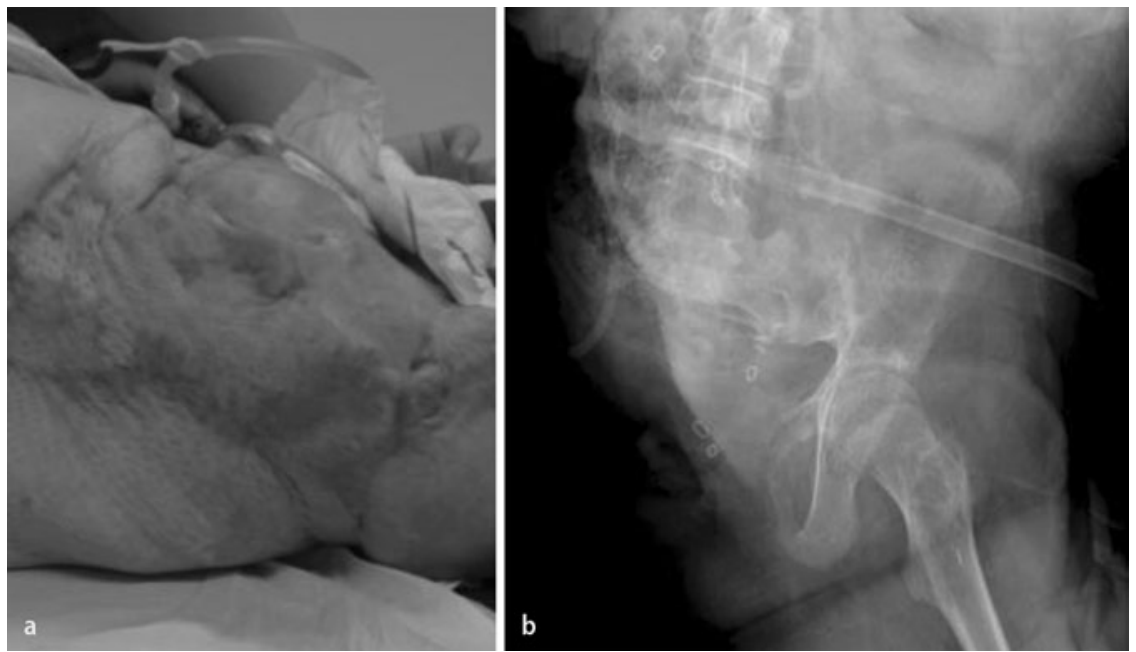

Abb. 7 A Fallbeispiel 3, 13 Jahre, weiblich, a Weichteiltrauma, b Hemipelvektomie

schen Untersuchung fand sich eine Beinheberschwäche der gesunden Seite, was für die weitere Versorgung erschwerend hinzukommt.

Operativ wurden durch den Abdominalchirurgen die beiden Stomas unterhalb des Rippenbogens angelegt; zudem liegt ein suprapubischer Katheter zur Ableitung des Urins ein. Inwiefern dies die prothetische Versorgung beeinträchtigen wird, ist derzeit noch nicht abzuschätzen.

Aufgrund dieser aktuellen Befunde konnte eine Versorgung bisher nicht eingeleitet werden. Im Gegensatz zum vorherigen Fallbeispiel fehlt hier das gesamte linke Beckenskelett, wodurch sich die Abstützfläche verringert. In der Regel sind die Beckenschaufel, der Sitz- oder Schambeinast als belastbare Fläche Voraussetzung zur Versorgung mit einem Beckenkorb. Nichtdestotrotz wird es möglich 
sein, eine prothetische Versorgung unter Einbeziehung der verbliebenen rechtsseitigen Beckenskelettanteile zeitnah einzuleiten.

\section{Fazit für die Praxis}

- Die Hilfsmittelversorgung bei Kindern und Jugendlichen ist ein wichtiger Baustein im gesamten Verlauf der Behandlung. Im Mittelpunkt stehen die Mobilität und Autonomie des Heranwachsenden. Mit den uns heute zur Verfügung stehenden Mitteln ist es möglich, den jungen Patienten Lebensqualität zurückzugeben.

- Durch die Weiterentwicklung von Operationstechniken und Materialien in der Orthopädietechnik sind individuelle Versorgungen möglich.

- Hier gilt es auch weiterhin anzuknüpfen, um den Versorgungsstandard für die Zukunft so hoch wie möglich halten zu können und den Kindern ein lebenswertes Dasein zu ermöglichen.

\section{Korrespondenzadresse}

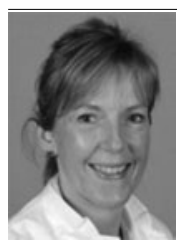

\section{S. Drisch}

Abteilung für BG-

Rehabilitation,

BG-Unfallklinik Murnau,

Prof.-Küntscher-Straße 8,

82418 Murnau

sabine.drisch@bgu-murnau.de

\section{Einhaltung ethischer Richtlinien}

Interessenkonflikt. S. Drisch, S. Simmel, V. Bühren und R. Mödl geben an, dass kein Interessenkonflikt besteht.

Dieser Beitrag beinhaltet keine Studien an Menschen oder Tieren.

The supplement containing this article is not sponsored by industry.

\section{Literatur}

1. Baumgartner R, Botta P, Bellmann D (2007) Amputation und Prothesenversorgung, 3. Aufl. Thieme, Stuttgart

2. Johnson CA (2003) Lower extremity amputations and prosthetics. Presented at Resident Lecture, Baltimore, March 27, 2003

3. Lambert CN (1964) The juvenile amputee. Arch Orthop Unfallchir 56:378-384
4. Neff G (1994) Amputationen im Wachstumsalter. Z Orthop Ihre Grenzgeb 132(3):227-234 